Actelion, Bayer, Boehringer Ingelheim, Bristol-Myers Squibb, Corbus Pharmaceuticals, Horizon Therapeutic, Galapagos, Roche/Genentech, GlaxoSmithKline, Mitsubishi Tanabe, Sanofi-Aventis/Genzyme, UCB

DOI: 10.1136/annrheumdis-2020-eular.3711

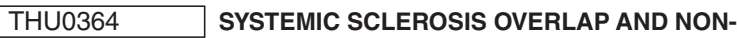 OVERLAP SYNDROMES SHARE SIMILAR CLINICAL CHARACTERISTICS BUT DRAMATICALLY DIFFERENT TREATMENT.}

M. Scherlinger ${ }^{1}$, J. Lutz ${ }^{2}$, J. Sibilia ${ }^{2}$, J. E. Gottenberg ${ }^{2}$, T. Schaeverbeke ${ }^{1}$, C. Richez ${ }^{1}$, E. Chatelus ${ }^{2}$, M. E. Truchetet ${ }^{1} .{ }^{1}$ C.H.U de Bordeaux, Rheumatology, Bordeaux, France; ${ }^{2}$ C.H.U de Strasbourg, Rheumatology, Strasbourg, France

Background: Overlap between systemic sclerosis (SSc) and another auto-immune systemic disease (AISD) in the same patient seems to be more frequent than each disease's prevalence would explain.

Objectives: Our aim was to investigate for overlap syndrome from 2 French cohorts of SSc patients and to compare their characteristics with non-overlap SSc.

Methods: Our study was retrospective observational and bicentric. Patients responding to the 2013 ACR-EULAR scleroderma classification criteria for SSc were screened for concomitant AISD. Patients satisfying 2010 ACR-EULAR diagnostic criteria for rheumatoid arthritis (RA) and/or 2016 ACR-EULAR classification criteria for Sjögren's syndrome (SgS) and/or 2012 SLICC systemic lupus erythematosus (SLE) classification criteria were included in our study. Patient, disease, and treatment characteristics were retrospectively retrieved from medical records and were compared to a SSc cohort.

Results: A population of 534 SSc patients was studied. Thirty-four (6.4\%) patients were identified as having overlap syndrome. There was $21(3.9 \%)$ patients with RA, 14 (2.6\%) with GSS and 4 (0.7\%) with SLE (5 patients had 2 AISD). Diagnosis of RA, SLE or SgS was made after diagnosis of SSc for $22(65 \%)$ patients, concomitantly for 10 patients $(29 \%)$, and before for 2 (6\%) patients. Interestingly, two patients with SSc/RA overlap were tested ACPA-positive 2 and 5 years before the first arthritis, respectively. Patients with SSc/RA were severe with $81 \%$ of them having erosive disease and despite treatment, only $48 \%(10 / 21)$ patients achieved RA remission (DAS28-CRP < 2.6) at the time of their last visit. Disease duration was longer in patients with SSc overlap syndrome compared to non-overlap patients $(15.5 \pm 10.6$ years vs. $9.5 \pm 8, p<0.001)$. Proportion of limited cutaneous SSc was similar in overlap and non-overlap groups $(70.6 \%$ vs. $75.5 \%$, respectively, $p=N S)$, as was the positivity for anti-centromeres antibodies (50\% vs. $43.2 \%$, respectively, $p=$ NS). The disease phenotype of SSc overlap syndrome was similar to the one of non-overlap SSc in terms of prevalence of pulmonary arterial hypertension, interstitial lung disease, digital ulcer and mortality. With respect to treatments, patients with overlap were more likely to receive glucocorticoids $(85.3 \% \mathrm{vs}$. $45 \%, p<0.001)$, immunosuppressive drugs $(82.4 \%$ vs. $49.2 \%, p<0.001)$ and biologic DMARD (bDMARD, $52.9 \%$ vs. $3.8 \%$, p < 0.001). The most prescribed bDMARDs in the overlap population was tocilizumab (40.6\%), TNF-alpha inhibitor (29.4\%) and rituximab (26.5\%) ( $p<0.001$ for all comparison vs. non-overlap SSc).

Conclusion: We found a prevalence of overlap syndrome higher than $5 \%$ among SSc patients. While SSc overlap and non-overlap share common characteristics, overlap patients are more likely to receive glucocorticoids and biologics such as anti-TNF. These overlap should be searched actively (eg, screening for ACPA) since some treatment used for other autoimmune diseases such as glucocorticoids or TNF-alpha inhibitor may be harmful in SSc.

Disclosure of Interests: Marc SCHERLINGER Consultant of: Amgen, Mylan, Fresenius Kabi, Johanna Lutz: None declared, Jean Sibilia: None declared, Jacques-Eric Gottenberg Grant/research support from: BMS, Pfizer, Consultant of: BMS, Sanofi-Genzyme, UCB, Speakers bureau: Abbvie, Eli Lilly and Co., Roche, Sanofi-Genzyme, UCB, Thierry Schaeverbeke: None declared, Christophe Richez Consultant of: Abbvie, Amgen, Mylan, Pfizer, Sandoz and UCB., Emmanuel Chatelus: None declared, Marie-Elise Truchetet: None declared

DOI: 10.1136/annrheumdis-2020-eular.3212

\section{THU0365 \\ INCREASED HSP9O IN MUSCLE TISSUE AND PLASMA ASSOCIATES WITH DISEASE ACTIVITY AND SKELETAL MUSCLE INVOLVEMENT IN PATIENTS WITH IDIOPATHIC INFLAMMATORY MYOPATHIES}

H. Štorkánová ${ }^{1,2}$, S. Oreska ${ }^{1,2}$, M. Špiritović ${ }^{3}$, B. Heřmánková ${ }^{3}$,

O. Kryštůfková ${ }^{1,2}$, H. Mann ${ }^{1,2}$, M. Komarc ${ }^{4}$, J. Zámečník ${ }^{5}$, K. Pavelka ${ }^{1,2}$, J. Vencovský ${ }^{1,2}$, L. Šenolt ${ }^{1,2}$, M. Tomcik ${ }^{1,2}{ }^{1}$ Institute of Rheumatology, Prague, Czech Republic; ${ }^{2}$ Department of Rheumatology, ${ }^{1}$ st Faculty of
Medicine, Charles University, Prague, Czech Republic, Prague, Czech Republic; ${ }^{3}$ Faculty of Physical Education and Sport, Charles University, Prague, Czech Republic, Department of Physiotherapy, Prague, Czech Republic; ${ }^{4}$ Department of Methodology, Faculty of Physical Education and Sport, Charles University, Prague, Czech Republic, Prague, Czech Republic; ${ }^{5}$ Department of Pathology and Molecular Medicine, 2nd Medical School and University Hospital Motol, Charles University, Prague, Czech Republic, Prague, Czech Republic

Background: Heat shock proteins (Hsps) are chaperones playing important roles in skeletal muscle physiology, adaptation to exercise or stress, and activation of inflammatory cells

Objectives: The aim of our study was to assess Hsp90 expression in muscle biopsies and plasma of patients with idiopathic inflammatory myopathies (IIM) and to characterize its association with IIM-related features.

Methods: Total of 277 patients with IIM (198 females, 79 males; mean age 54.8; disease duration 4.1 years; DM, 104/PM, 108/CADM, 31/IMNM, 25) and 157 healthy individuals (92 females, 65 males; mean age 47.0) were included in plasma analysis. Muscle biopsy samples (PM, DM, IMNM, myodystrophy, myasthenia gravis) were stained for Hsp90a (Thermo Fisher Scientific, USA) and Hsp90 $\beta$ (Abcam, UK). Plasma Hsp90 was measured by ELISA kit (eBioscience, Vienna, Austria). The cytokines/chemokines were analysed by using BioPlex Pro ${ }^{\mathrm{TM}}$ human Cytokine 27-plex Assay (BIO-RAD, California, USA.Data are presented as median(IQR).

Results: In muscle biopsies, Hsp90 expression of both subunits (alpha and beta) was higher in IIM than in controls. Increased Hsp90 was detected in perifascicular degenerating and regenerating fibers, inflammatory cells (DM, PM), and necrotic and regenerating fibers (IMNM). Plasma Hsp90 levels were increased in IIM patients compared to healthy controls $(55.9(46.9-62.5)$ vs 9.76(7.5 - 13.8), $\mathrm{p}<0.0001)$, and in individual subgroups of IIM vs. healthy controls (DM-22.01(14.1 - 41.2), PM-19.7(14.3 - 42.2), CADM-18.9(11.7 - 29.7), IMNM-19.6(16.3 - 45.5), p<0.0001 for all). Hsp90 was higher in males compared to females $(p=0.040)$ and in patients with ILD $(p=0.003)$, cardiac involvement $(p=0.004)$, dysphagia $(p=0.018)$ and presence of anti-Ro52 $(p=0.036)$. Hsp90 levels in all patients positively correlated with muscle enzymes (Tab.1). Hsp90 was associated with disease activity and skeletal muscle involvement (Tab.1). Out of all clinical parameters listed in above-mentioned univariate analysis, in multiple regression analysis Hsp90 levels in IIM patients were significantly affected by muscle enzymes only $(p<0.0001, \beta=0.345)$. Furthermore, Hsp90 positively correlated with some crucial cytokines involved in pathogenesis of myositis (Tab. 1).

Tab. 1.

\begin{tabular}{lll}
\hline Clinical parameters & Spearman's $\mathbf{r}$ & $\mathbf{p}$-value \\
\hline LDH; AST; ALT & $0.554 ; 0.383 ; 0.181$ & $<0.0001 ;<$ \\
& & $0.0001 ; 0.003$ \\
& $0.223 ; 0.217 ; 0.175 ; 0.159$ & $\begin{array}{l}<0.001 ;<0.001 ; \\
0.004 ; 0.012\end{array}$ \\
PtDGA; PhDGA; MITAX; MYOACT & & 0.001 \\
& 0.201 & 0.018 \\
Pulmonary disease activity & 0.146 & $0.042 ; 0.043 ;$ \\
Muscle disease activity & $-0.126 ;-0.125 ;-0.159 ;-0.143$ & $0.011 ; 0.023$ \\
MMT8, total score; m. biceps brachii; m. & & 0.041 \\
gluteus maximus; m. iliopsoas & 0.150 & 0.006 \\
MDI - Myositis damage index - severity & 0.183 & $0.002 ;<0.0001 ;$ \\
Current Prednisone equivalent dose & $0.188 ; 0.269 ; 0.190 ; 0.182 ;$ & $0.002 ; 0.003 ;<$ \\
Cytokines: IL-1b; IL-2; IL-4; IL-6; IFN- $\gamma$ & 0.229 & 0.0001 \\
& &
\end{tabular}

Conclusion: We demonstrate increased $\mathrm{Hsp} 90$ expression in IIM muscle biopsy samples, specifically in inflammatory cells, degenerating, regenerating and/or necrotic fibers. Increased Hsp90 plasma levels in IIM patients are associated with disease activity and damage, and with the involvement of proximal skeletal muscles, heart and lungs.

Acknowledgments: Supported by AZV-16-33542A, MHCR 023728 and SVV $-260373$.

Disclosure of Interests: Hana Štorkánová: None declared, Sabina Oreska: None declared, Maja Špiritović: None declared, Barbora Heřmánková: None declared, Olga Kryštůfková: None declared, Heřman Mann: None declared, Martin Komarc: None declared, Josef Zámečník: None declared, Karel Pavelka Consultant of: Abbvie, MSD, BMS, Egis, Roche, UCB, Medac, Pfizer, Biogen, Speakers bureau: Abbvie, MSD, BMS, Egis, Roche, UCB, Medac, Pfizer, Biogen, Jiří Vencovský: None declared, Ladislav Šenolt: None declared, Michal Tomcik: None declared

DOI: 10.1136/annrheumdis-2020-eular.2746 